\title{
A new explicit formula for the Bernoulli and Genocchi numbers in terms of the Stirling numbers
}

\author{
Bai-Ni Guo $^{1, *}$, Feng Qi ${ }^{2,3}$ \\ ${ }^{1}$ School of Mathematics and Informatics, Henan Polytechnic University, Jiaozuo City, Henan Province, 454010, China \\ 2 College of Mathematics, Inner Mongolia University for Nationalities, \\ Tongliao City, Inner Mongolia Autonomous Region, 028043, China \\ 3 Department of Mathematics, College of Science, Tianjin Polytechnic University, Tianjin City, 300387, China \\ *Corresponding author's e-mail: bai.ni.guo@gmail.com,bai.ni.guo@hotmail.com \\ * Corresponding author's URL: http: // www. researcherid. com/ rid/C-8032-2013
}

Copyright (c)2015 Bai-Ni Guo and Feng Qi. This is an open access article distributed under the Creative Commons Attribution License, which permits unrestricted use, distribution, and reproduction in any medium, provided the original work is properly cited.

\begin{abstract}
In the paper, the authors concisely review some explicit formulas and establish a new explicit formula for the Bernoulli and Genocchi numbers in terms of the Stirling numbers of the second kind.
\end{abstract}

Keywords: explicit formula; Bernoulli number; Genocchi number; Stirling number of the second kind

MSC: Primary 11B68; Secondary $11 B 73$

\section{Introduction and main results}

It is well known that the Bernoulli numbers $B_{n}$ for $n \geq 0$ may be defined by the power series expansion

$\frac{x}{e^{x}-1}=\sum_{n=0}^{\infty} B_{n} \frac{x^{n}}{n !}=1-\frac{x}{2}+\sum_{k=1}^{\infty} B_{2 k} \frac{x^{2 k}}{(2 k) !}, \quad|x|<2 \pi$

that Euler polynomials $E_{n}(x)$ are defined by

$\frac{2 e^{x t}}{e^{t}+1}=\sum_{n=0}^{\infty} E_{n}(x) \frac{t^{n}}{n !}$,

that the Genocchi numbers $G_{n}$ for $n \in \mathbb{N}$ are given by the generating function

$\frac{2 t}{e^{t}+1}=\sum_{n=1}^{\infty} G_{n} \frac{t^{n}}{n !}$, 
and that the Stirling numbers of the second kind which may be generated by

$\frac{\left(e^{x}-1\right)^{k}}{k !}=\sum_{n=k}^{\infty} S(n, k) \frac{x^{n}}{n !}, \quad k \in \mathbb{N}$

and may be computed by

$S(k, m)=\frac{1}{m !} \sum_{\ell=1}^{m}(-1)^{m-\ell}\left(\begin{array}{c}m \\ \ell\end{array}\right) \ell^{k}, \quad 1 \leq m \leq k$.

By the way, the Stirling number of the second kind $S(n, k)$ may be interpreted combinatorially as the number of ways of partitioning a set of $n$ elements into $k$ nonempty subsets.

The Bernoulli numbers $B_{n}$ for $n \in\{0\} \cup \mathbb{N}$ satisfy

$B_{0}=1, \quad B_{1}=-\frac{1}{2}, \quad B_{2 n+2} \neq 0, \quad B_{2 n+3}=0$.

For $n \in \mathbb{N}$, the Genocchi numbers meet $G_{2 n+1}=0$. The first few Genocchi numbers $G_{n}$ are listed in Table 1.1. The

Table 1.1: The first few Genocchi numbers $G_{n}$

\begin{tabular}{|c|c|c|c|c|c|c|c|c|c|c|}
\hline$n$ & 1 & 2 & 4 & 6 & 8 & 10 & 12 & 14 & 16 & 18 \\
\hline$G_{n}$ & 1 & -1 & 1 & -3 & 17 & -155 & 2073 & -38227 & 929569 & -28820618 \\
\hline
\end{tabular}

Genocchi numbers $G_{2 n}$ may be represented in terms of the Bernoulli numbers $B_{2 n}$ and Euler polynomials $E_{2 n-1}(0)$ as

$G_{2 n}=2\left(1-2^{2 n}\right) B_{2 n}=2 n E_{2 n-1}(0), \quad n \in \mathbb{N}$.

See $[1$, p. 49]. As a result, we have

$G_{n}=2\left(1-2^{n}\right) B_{n}, \quad n \in \mathbb{N}$.

The first formula for the Bernoulli numbers $B_{n}$ listed in [2] is

$B_{n}=\sum_{k=0}^{n} \frac{1}{k+1} \sum_{j=0}^{k}(-1)^{j}\left(\begin{array}{l}k \\ j\end{array}\right) j^{n}, \quad n \geq 0$,

which is a special case of the general formula $[13,(2.5)]$. The formula (9) is equivalent to

$B_{n}=\sum_{k=0}^{n}(-1)^{k} \frac{k !}{k+1} S(n, k), \quad n \in\{0\} \cup \mathbb{N}$,

which was listed in [3, p. 536] and [4, p. 560]. Recently, four alternative proofs of the formula (10) were provided in $[7,16]$. A generalization of the formula (10) was supplied in [6]. In all, we may collect at least seven alternative proofs for the formula (9) or (10) in $[2,4,7,13,14,16]$ and closely related references therein.

In $[2$, p. $48,(11)]$, it was deduced that

$B_{n}=\sum_{j=0}^{n}(-1)^{j}\left(\begin{array}{c}n+1 \\ j+1\end{array}\right) \frac{n !}{(n+j) !} \sum_{k=0}^{j}(-1)^{j-k}\left(\begin{array}{l}j \\ k\end{array}\right) k^{n+j}, \quad n \geq 0$

which may be rearranged as

$B_{n}=\sum_{i=0}^{n}(-1)^{i} \frac{\left(\begin{array}{c}n+1 \\ i+1\end{array}\right)}{\left(\begin{array}{c}n+i \\ i\end{array}\right)} S(n+i, i), \quad n \geq 0$.

The formula (12) was rediscovered in the paper [8]. On 21 January 2014, the authors searched out that the formula (12) was also derived in [12, p. 59] and [17, p. 140]. 
In [11, p. 1128, Corollary], among other things, it was found that

$B_{2 k}=\frac{1}{2}-\frac{1}{2 k+1}-2 k \sum_{i=1}^{k-1} \frac{A_{2(k-i)}}{2(k-i)+1}$

for $k \in \mathbb{N}$, where $A_{m}$ is defined by

$\sum_{m=1}^{n} m^{k}=\sum_{m=0}^{k+1} A_{m} n^{m}$

In [15, Theorem 1.4], among other things, it was presented that

$B_{2 k}=\frac{(-1)^{k-1} k}{2^{2(k-1)}\left(2^{2 k}-1\right)} \sum_{i=0}^{k-1} \sum_{\ell=0}^{k-i-1}(-1)^{i+\ell}\left(\begin{array}{c}2 k \\ \ell\end{array}\right)(k-i-\ell)^{2 k-1}, \quad k \in \mathbb{N}$.

In [10, Theorem 3.1], it was obtained that

$B_{2 k}=1+\sum_{m=1}^{2 k-1} \frac{S(2 k+1, m+1) S(2 k, 2 k-m)}{\left(\begin{array}{c}2 k \\ m\end{array}\right)}-\frac{2 k}{2 k+1} \sum_{m=1}^{2 k} \frac{S(2 k, m) S(2 k+1,2 k-m+1)}{\left(\begin{array}{c}2 k \\ m-1\end{array}\right)}, k \in \mathbb{N}$.

The aim of this paper is to find the following new explicit formula for the Bernoulli numbers $B_{k}$, or say, the Genocchi numbers $G_{k}$, in terms of the Stirling numbers of the second kind $S(k, m)$.

Theorem 1.1 For all $k \in \mathbb{N}$, the Genocchi numbers $G_{k}$ may be computed by

$G_{k}=2\left(1-2^{k}\right) B_{k}=(-1)^{k} k \sum_{m=1}^{k}(-1)^{m} \frac{(m-1) !}{2^{m-1}} S(k, m)$.

\section{Proof of Theorem 1.1}

Differentiating on both sides of the equation (3) and employing Leibniz identity for differentiation give

$\left(\frac{2 t}{e^{t}+1}\right)^{(k)}=2\left[t\left(\frac{1}{e^{t}+1}\right)^{(k)}+k\left(\frac{1}{e^{t}+1}\right)^{(k-1)}\right]=\sum_{n=k}^{\infty} G_{n} \frac{t^{n-k}}{(n-k) !}$.

In [9, Theorem 2.1] and [18, Theorem 3.1], it was obtained that, when $\lambda>0$ and $t \neq-\frac{\ln \lambda}{\alpha}$ or when $\lambda<0$ and $t \in \mathbb{R}$,

$\left(\frac{1}{\lambda e^{\alpha t}-1}\right)^{(k)}=(-1)^{k} \alpha^{k} \sum_{m=1}^{k+1}(m-1) ! S(k+1, m)\left(\frac{1}{\lambda e^{\alpha t}-1}\right)^{m}$.

Specially, when $\lambda=-1$ and $\alpha=1$, the identity (17) becomes

$\left(\frac{1}{e^{t}+1}\right)^{(k)}=(-1)^{k+1} \sum_{m=1}^{k+1}(-1)^{m}(m-1) ! S(k+1, m)\left(\frac{1}{e^{t}+1}\right)^{m}$.

Consequently, it follows that

$$
\begin{aligned}
& G_{k}=\lim _{t \rightarrow 0} \sum_{n=k}^{\infty} G_{n} \frac{t^{n-k}}{(n-k) !}=2 k \lim _{t \rightarrow 0}\left(\frac{1}{e^{t}+1}\right)^{(k-1)} \\
& =2 k(-1)^{k} \sum_{m=1}^{k}(-1)^{m}(m-1) ! S(k, m) \lim _{t \rightarrow 0}\left(\frac{1}{e^{t}+1}\right)^{m} \\
& =(-1)^{k} k \sum_{m=1}^{k}(-1)^{m} \frac{(m-1) !}{2^{m-1}} S(k, m) .
\end{aligned}
$$

The proof of Theorem 1.1 is complete.

Remark 2.1 This paper is a slightly modified version of the preprint [5]. 


\section{References}

[1] L. Comtet, Advanced Combinatorics: The Art of Finite and Infinite Expansions, Revised and Enlarged Edition, D. Reidel Publishing Co., Dordrecht and Boston, 1974.

[2] H. W. Gould, Explicit formulas for Bernoulli numbers, Amer. Math. Monthly 79 (1972), 44-51.

[3] R. L. Graham, D. E. Knuth, and O. Patashnik, Concrete Mathematics-A Foundation for Computer Science, Addison-Wesley Publishing Company, Advanced Book Program, Reading, MA, 1989.

[4] R. L. Graham, D. E. Knuth, and O. Patashnik, Concrete Mathematics-A Foundation for Computer Science, 2nd ed., Addison-Wesley Publishing Company, Reading, MA, 1994.

[5] B.-N. Guo and F. Qi, A new explicit formula for Bernoulli and Genocchi numbers in terms of Stirling numbers, available online at http://arxiv.org/abs/1407.7726.

[6] B.-N. Guo, I. Mezö, and F. Qi, An explicit formula for Bernoulli polynomials in terms of r-Stirling numbers of the second kind, available online at http://arxiv.org/abs/1402.2340.

[7] B.-N. Guo and F. Qi, Alternative proofs of a formula for Bernoulli numbers in terms of Stirling numbers, Analysis (Berlin) 34 (2014), no. 2, 187-193; Available online at http://dx.doi.org/10.1515/anly-2012-1238.

[8] B.-N. Guo and F. Qi, An explicit formula for Bernoulli numbers in terms of Stirling numbers of the second kind, J. Anal. Number Theory 3 (2015), no. 1, 27-30; Available online at http://dx.doi.org/10.12785/ jant/030105.

[9] B.-N. Guo and F. Qi, Explicit formulae for computing Euler polynomials in terms of Stirling numbers of the second kind, J. Comput. Appl. Math. 272 (2014), 251-257; Available online at http://dx.doi.org/10.1016/ j.cam.2014.05.018.

[10] B.-N. Guo and F. Qi, Some identities and an explicit formula for Bernoulli and Stirling numbers, J. Comput. Appl. Math. 255 (2014), 568-579; Available online at http://dx.doi.org/10.1016/j.cam.2013.06.020.

[11] S.-L. Guo and F. Qi, Recursion formulae for $\sum_{m=1}^{n} m^{k}$, Z. Anal. Anwendungen 18 (1999), no. 4, 1123-1130; Available online at http://dx.doi.org/10.4171/ZAA/933.

[12] S. Jeong, M.-S. Kim, and J.-W. Son, On explicit formulae for Bernoulli numbers and their counterparts in positive characteristic, J. Number Theory 113 (2005), no. 1, 53-68; Available online at http://dx.doi.org/ $10.1016 / j \cdot j n t .2004 .08 .013$.

[13] J. Higgins, Double series for the Bernoulli and Euler numbers, J. London Math. Soc. 2nd Ser. 2 (1970), 722-726; Available online at http://dx.doi.org/10.1112/jlms/2.Part_4.722.

[14] B. F. Logan, Polynomials related to the Stirling numbers, AT\&T Bell Laboratories internal technical memorandum, August 10, 1987.

[15] F. Qi, Derivatives of tangent function and tangent numbers, available online at http://arxiv. org/abs/1202. 1205.

[16] F. Qi and B.-N. Guo, Alternative proofs of a formula for Bernoulli numbers in terms of Stirling numbers, Analysis (Berlin) 34 (2014), no. 3, 311-317; Available online at http://dx.doi.org/10.1515/anly-2014-0003.

[17] S. Shirai and K.-I. Sato, Some identities involving Bernoulli and Stirling numbers, J. Number Theory 90 (2001), no. 1, 130-142; Available online at http://dx.doi.org/10.1006/jnth.2001.2659.

[18] A.-M. Xu and Z.-D. Cen, Some identities involving exponential functions and Stirling numbers and applications, J. Comput. Appl. Math. 260 (2014), 201-207; Available online at http://dx.doi.org/10.1016/j.cam. 2013. 09.077. 